\title{
Evaluación del Recurso Hidrocinético en las Partes Bajas de la Región Hidrológica 29Ba de la Cuenca del Río Coatzacoalcos
}

\section{Hydrokinetic Resource Assessments in the Lower Parts of the 29Ba Hydrological Region of the Coatzacoalcos River Basin}

\author{
ESPINOSA-ARENAL Francisco*†, PEREA-ALCALÁ, Gerardo, ÁVILA-HERNÁNDEZ, José Angel \\ y DELFÍN-ANZURES, Rachel
}

Facultad de Ingeniería, Universidad Veracruzana, Av. Universidad km. 7.5, Coatzacoalcos, Veracruz 96538, México. Centro de Investigación en Recursos Energéticos y Sustentables, Av. Universidad km. 7.5, Coatzacoalcos, Veracruz 96538 , México. ID $1^{\mathrm{er}}$ Autor: Francisco, Espinosa-Arenal / ORC ID: 0000-0002-3800-757X, Researcher ID Thomson: R-4728-2018, CVU
CONACYT ID: 45862

ID $1^{\text {er }}$ Coautor: Gerardo, Alcalá-Perea / ORC ID: 0000-0003-2186-2323, Researcher ID Thomson: O-7148-2018, CVU CONACYT ID: 270995

ID $2^{\text {do }}$ Coautor: José Angel Hernández-Ávila / ORC ID: 0000-0001-5642-9533, CVU CONACYT ID: 998863

ID $3^{\text {er }}$ Coautor: Rachel, Delfin-Anzures / ORC ID: 0000-0002-5116-7172, CVU CONACYT ID: 998828

DOI: $10.35429 / J C E .2019 .7 .3 .1 .6$

Recibido 03 de Enero, 2019; Aceptado 30 Marzo, 2019

\begin{abstract}
Resumen
La energía hidrocinética es inducida por la velocidad en los causes naturales de los ríos y es utilizada para la generación eléctrica. Los asentamientos humanos siempre se han establecidos en las márgenes de los ríos, por lo tanto, resulta de mucha utilidad determinar el potencial hidrocinético en los sitios donde haya poblaciones a la orilla de los ríos, principalmente en los lugares donde no se cuenta con el servicio de energía eléctrica. En este trabajado se evalúa el recurso hidrocinético en diferentes poblados de las partes planas de la región hidrológica 29Ba. Los caudales en los ríos son calculados tomando en cuenta el uso de suelo, la precipitación de distintas estaciones meteorológicas, los cuales son procesados en un Sistema de Información Geográfica (SIG). Los caudales calculados son comparados con las mediciones que se realizaron en la estación hidrométrica Las perlas de la CONAGUA. Las velocidades a lo largo del río son calculadas con el modelo numérico Hec-Ras, posteriormente se estima el potencial hidrocinético mensual mínimo, máximo y promedio con algunos modelos de turbinas diseñadas para este fin.
\end{abstract}

Energía Hidrocinética, SIG, Hec-Ras

\begin{abstract}
Hydrokinetic energy is induced by the velocity in the natural causes of rivers and is used for electric generation. Human settlements have always been established on the margins of the rivers, therefore it is very useful to evaluate the hydrokinetic resource in the places where there are populations on the banks of the rivers, mainly in places where there is no service available electric power. In this work, the hydrokinetic potential along the river in different sites of the flat parts of the hydrological region 29Ba is determined. The flows in the rivers are calculated taking into account the use of soil, the precipitation of different meteorological stations, which are processed in a Geographic Information System (GIS). The calculated flows are compared with the observed data of the hydrometric station Las Perlas of CONAGUA. The velocities along the river are calculated with the numeric model Hec-Ras, later the minimum, maximum and average monthly hydrokinetic potential is estimated with some turbine models designed for this purpose.
\end{abstract}

Hydrokinetic energy, SIG, Hec-Ras

Citación: ESPINOSA-ARENAL Francisco, PEREA-ALCALÁ, Gerardo, ÁVILA-HERNÁNDEZ, José Angel y DELFÍNANZURES, Rachel. Evaluación del Recurso Hidrocinético en las Partes Bajas de la Región Hidrológica 29Ba de la Cuenca del Río Coatzacoalcos. Revista de Ingeniería Civil. 2019. 3-7: 1-6

\footnotetext{
* Correspondencia del Autor (correo electrónico: franciespinosa@uv.mx)

$\dagger$ Investigador contribuyendo como primer autor.
} 


\section{Introducción}

Las energías renovables juegan un papel muy importante en la generación de energía eléctrica. El incremento constante del consumo de energía en todo el mundo, junto la atenuación del cambio climático, han forzado a que se tome en cuenta una mayor participación de las energías renovables (SENER, 2017). La generación de energía eléctrica utilizando medios convencionales, como los combustibles fósiles, ha generado severos problemas ambientales (Solomon et al, 2007). Por lo tanto, es conveniente el uso de las energías limpias, en la gran mayoría de los países incluyendo a México. El estudio y desarrollo para la producción de electricidad por medio de las energías renovables están concentradas en la energía eólica y solar mayormente.

Para el periodo del 2007 al 2017 tuvieron un crecimiento promedio anual del $47.6 \%$ y 43.2\% respectivamente (SENER, 2018). La energía cinética que posee el flujo de agua en movimiento puede aprovechar tres tipos de recursos, ríos en tierra adentro, mareas con estuarios y las corrientes marinas. Este tipo de energía pertenece al campo de las hidroeléctricas (Lata, 2019). Aunque la energía hidroeléctrica es la que tiene una mayor participación con el $65 \%$ generación eléctrica en México, la energía hidrocinética no está incluida en estas estadísticas. Se ha estudiado que la explotación de energía hidrocinética tiene un bajo impacto ambiental, esto podría aumentar la producción de energía principalmente en los países en desarrollo para seguir con su crecimiento (da Silva et. al, 2017).

En el Mapa de Ruta Tecnológica en energía del Océano de la SENER (2017b) se dan a conocer los mapas de corrientes marinas del Océano pacífico, Golfo de México y mar Caribe del verano e invierno, con el propósito de impulsar turbinas para generación de energía eléctrica. Punys et. al (2015) realiza el estudio teórico del potencial hidrocinético obteniendo las velocidades en el segundo río más importante de Lituania con un modelo unidimensional y utilizando varias turbinas. Motoya et. Al (2016), realiza un estudio similar en los canales de descarga de una central hidroeléctrica en Colombia, obteniendo las velocidades con un modelo unidimensional y utilizando varias turbinas.
Álvarez et. al (2016) utiliza prácticamente la metodología de los autores anteriores para analizar el recurso hidrocinético en la desembocadura del río Nalón. Las turbinas para el aprovechamiento del recurso hidrocinético, existen ya una gama de turbinas comerciales y proyectos de investigación, Motoya et. al (2016) utiliza hasta 4 turbinas diferentes, donde la velocidad mínima nominal para generación eléctrica de $1.5 \mathrm{~m} / \mathrm{s}$. Arrieta et. al (2019) realiza una investigación numérica sobre una turbina hidrocinética de eje horizontal. Peña (2013) realiza un diseño numérico experimental par una turbina hidrocinética para ríos pocos caudalosos. Sørnes (2010) hace un análisis de distintas turbinas comerciales, donde destaca turbinas donde tienen una producción de energía eléctrica desde una velocidad de $0.5 \mathrm{~m} / \mathrm{s}$. Con las investigaciones anteriores será posibles estimar el recurso hidrocinético siempre y cuando se puedan determinar las velocidades.

\section{Área de estudio}

México cuenta con 32 regiones hídricas, la cuenca del Coatzacoalcos es la tercera más grande del país, está situada en la región Hidrológico-Administrativa X Golfo Centro o región h29, con un escurrimiento natural medio superficial de $28717.4 \mathrm{hm}^{3} / \mathrm{año}$, con un área de $21,336 \mathrm{~km}^{2}$ y una precipitación media a anual de 1,626 mm (CONAGUA, 2018). Las subcuencas que conforman el Consejo de cuenca de Coatzacoalcos son: RH29Aa a RH29Ah, RH29Ba a RH29Bm (CONAGUA, 2014). En este trabajo se analiza solo la región RH29Ba, también conocida como el alto río Coatzacoalcos, en esta zona se encuentra ubicada la estación hidrométrica Las perlas (DOF, 2017).

Las comunidades más importantes donde se hace el estudio del potencial hidrocinético son: Las Perlas, Jesús Carranza y Suchilapan, debido a la cercanía del flujo del río. La cuenca Alto Río Coatzacoalcos, drena una superficie de 10,652.5 kilómetros cuadrados; se encuentra ubicada entre las coordenadas geográficas $17^{\circ} 32^{\prime} 26^{\prime \prime}$ y $16^{\circ} 37^{\prime} 52^{\prime \prime}$ de Latitud Norte y entre $95^{\circ} 44^{\prime} 58^{\prime \prime}$ y $94^{\circ} 11^{\prime} 01^{\prime \prime}$ de Longitud Oeste. 
Está delimitada al norte por la cuenca hidrológica Bajo Río Coatzacoalcos, al sur con la Región Hidrológica Número 22 Tehuantepec, al este con la cuenca hidrológica Alto Río Uxpanapa y la Región Hidrológica Número 30 Grijalva-Usumacinta y al oeste por la Región Hidrológica Número 28 Papaloapan. La longitud de su cauce principal es de aproximadamente 246 kilómetros (DOF, 2017).

En la figura 1 se observa la delimitación del área de aportación $\left(8,915 \mathrm{~km}^{2}\right)$ a la estación hidrométrica las perlas (comunidad de estudio del mismo nombre) y los sitios de estudio Jesús Carranza y Suchilapan, con un área de escurrimiento de $3,337 \mathrm{~km}^{2}$ y $5,578 \mathrm{~km}^{2}$ respectivamente. Los caudales fueron calculados con la ayuda del Sistema de Información Geografía (SIG) QGIS 3.4.8 (QGIS, 2018). En este modelo se procesa las imágenes de satélite de 30m de resolución (Alaska Satellite Facility, 2011) para delimitar el área de influencia de escurrimiento.

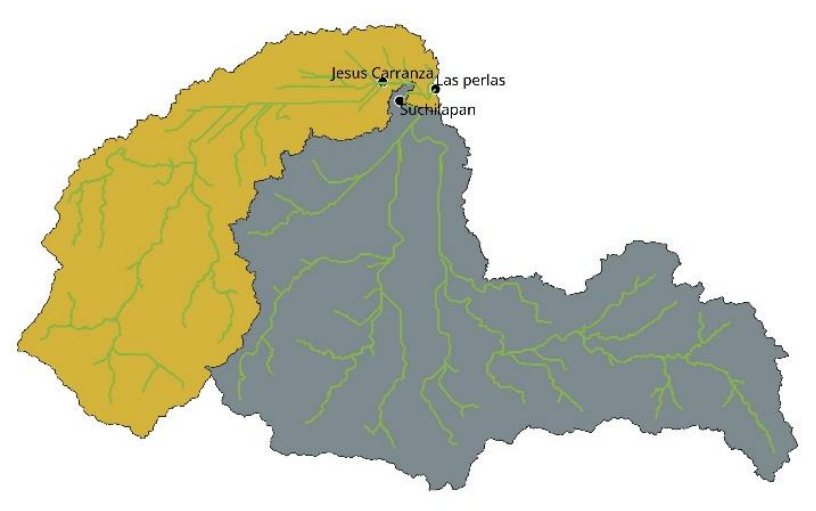

Figura 1 Áreas y sitios de estudio

En la Figura 2 se muestran las estaciones climatológicas de la CONAGUA (donde se obtiene la precipitación media por mes del año 1962 hasta el 2015), se observa también las isoyetas del mes de septiembre, en esta figura se muestra la escala del coeficiente de escurrimiento, el cual fue posible calcular con el uso de suelo y vegetación (INEGI, 2015) y edafología (INEGI, 2013). Posteriormente con el método racional son calculados los caudales mensuales, los cuales son comparados con los datos medidos de la estación hidrométrica Las Perlas. Con el modelo Hec-Ras (US Army Corps of Engineers. Hydrologic Engineering Center; 2017) versión 5.0.7 y un modelo de elevación digital de $12.5 \mathrm{~m}$ y los caudales calculados, se determinan las velocidades en los tres sitios que se muestran la la figura 1. En la figura 3 se puede observar las secciones del río en el sitio
Las perlas donde se calcularon las velocidades, utilizando el coeficiente de Manning y la pendiente del cauce natural del río.

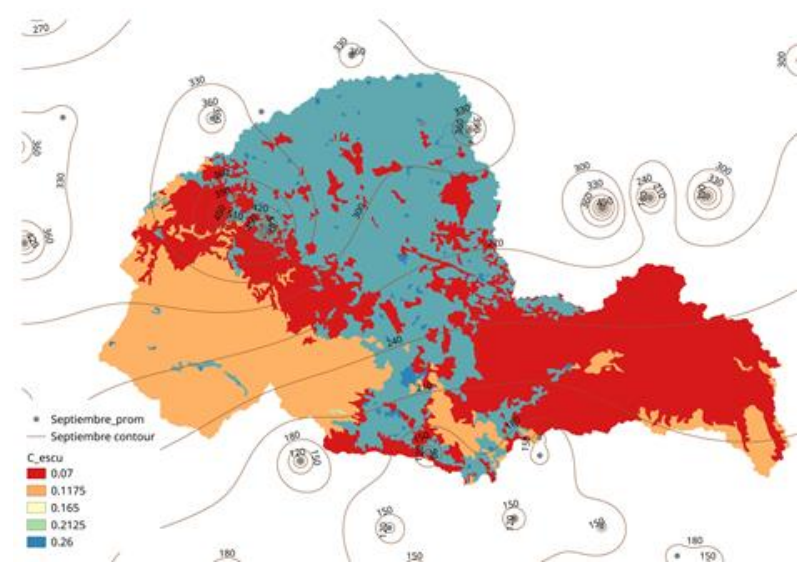

Figura 2 Mapa de isoyetas, estaciones meteorológicas y coeficientede escurrimiento

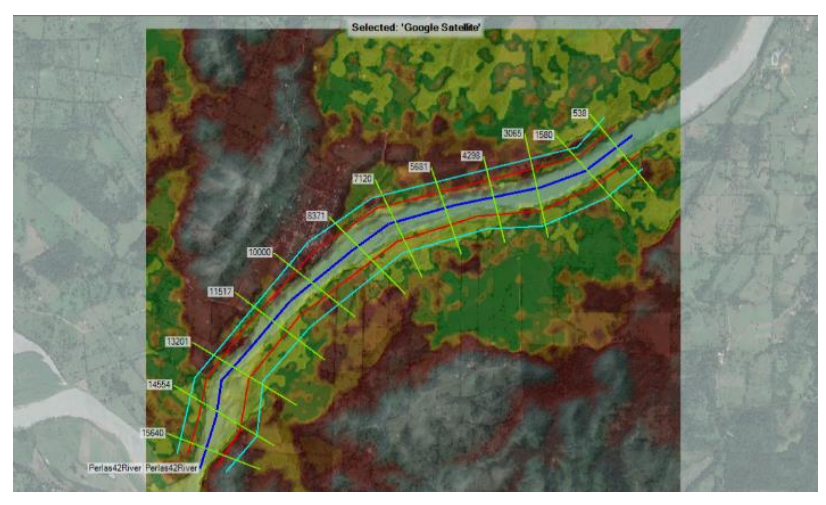

Figura 3 Secciones del río en el sitio las sitio Las Perlas modelado con Hec-Ras.

\section{Cálculo de Caudales}

En la Tabla 1, se muestran los valores de los caudales promedio historicos 1953-1973, 1975$1976,1978,1981-1985,1987-2006$ en la estación hidrométrica Las Perlas (Comisión Nacional del Agua) de los 12 meses del año.

\begin{tabular}{|l|r|r|r|}
\hline \multicolumn{1}{|c|}{ Mes } & Qobs. $\left(\mathbf{m}^{\mathbf{3}} \mathbf{s} \mathbf{s}\right)$ & Qcalc. $\left(\mathbf{m}^{\mathbf{3}} \mathbf{s}\right)$ & Error $\mathbf{( \% )}$ \\
\hline Enero & 285.31 & 265.72 & 6.9 \\
\hline Febrero & 225.88 & 202.73 & 10.2 \\
\hline Marzo & 165.53 & 136.31 & 17.6 \\
\hline Abril & 124.8 & 111.42 & 10.7 \\
\hline Mayo & 116.36 & 142.00 & -22.0 \\
\hline Junio & 242.96 & 251.26 & -3.4 \\
\hline Julio & 718.89 & 753.07 & -4.8 \\
\hline Agosto & 906.79 & 919.035 & -1.4 \\
\hline Septiembre & 1107.8 & 1144.81 & -3.3 \\
\hline Octubre & 819.37 & 832.457 & -1.6 \\
\hline Noviembre & 477.13 & 314.18 & 34.2 \\
\hline Diciembre & 363.46 & 476.08 & -31.0 \\
\hline
\end{tabular}

Tabla 1 Caudales de la estación Las Perlas observados, calculados y error porcentual.

ESPINOSA-ARENAL Francisco, PEREA-ALCALÁ, Gerardo, ÁVILAHERNÁNDEZ, José Angel y DELFÍN-ANZURES, Rachel. Evaluación del Recurso Hidrocinético en las Partes Bajas de la Región Hidrológica 29Ba de la Cuenca del Río Coatzacoalcos. Revista de Ingeniería Civil. 2019 
Una vez validado el modelo hidrológico comparando los caudales (tabla 1) se precedo a calcular los caudales donde no existe medición, es decir en el sitio Jesús Carranza y Suchilapan mostrados en la figura 1. El método de cálculo de caudales es el mismo que se describe en la sección 1. En la tabla 2 se muestran los valores de caudales de los sitios antes mencionados.

\begin{tabular}{|l|r|r|}
\hline \multicolumn{1}{|c|}{ Mes } & \multicolumn{1}{c|}{$\begin{array}{c}\text { Qcalc. }\left(\mathrm{m}^{3} / \mathrm{s}\right) . \\
\text { Jesús Carranza }\end{array}$} & $\begin{array}{c}\text { Qcalc. }\left(\mathrm{m}^{3} / \mathrm{s}\right) . \\
\text { Suchilapan }\end{array}$ \\
\hline Enero & 99.5 & 166.3 \\
\hline Febrero & 75.9 & 126.8 \\
\hline Marzo & 51.0 & 85.3 \\
\hline Abril & 41.7 & 69.7 \\
\hline Mayo & 53.2 & 88.8 \\
\hline Junio & 94.1 & 157.2 \\
\hline Julio & 281.9 & 471.2 \\
\hline Agosto & 344.0 & 575.0 \\
\hline Septiembre & 428.5 & 716.3 \\
\hline Octubre & 311.6 & 520.8 \\
\hline Noviembre & 117.6 & 196.6 \\
\hline Diciembre & 178.2 & 297.9 \\
\hline
\end{tabular}

Tabla 2 Caudales calculados del sitio Jesús Carranza

\section{Cálculo de velocidades}

En la figura 4 se muestra la simulación del flujo con el modelo Hec-Ras en la sección 10000 del río (ver figura 3), durante el mes de septiembre, la simulación fue realizada con el caudal de $1144.81 \mathrm{~m}^{3} / \mathrm{s}$ como se muestra en la tabla 1 . Se elije esta sección debido a que es la parte más cerca a la comunidad Las Perlas (mismo sitio que la estación hidrométrica del mismo nombre). En la Figura 4 se observa el perfil, ancho y profundidad del río.

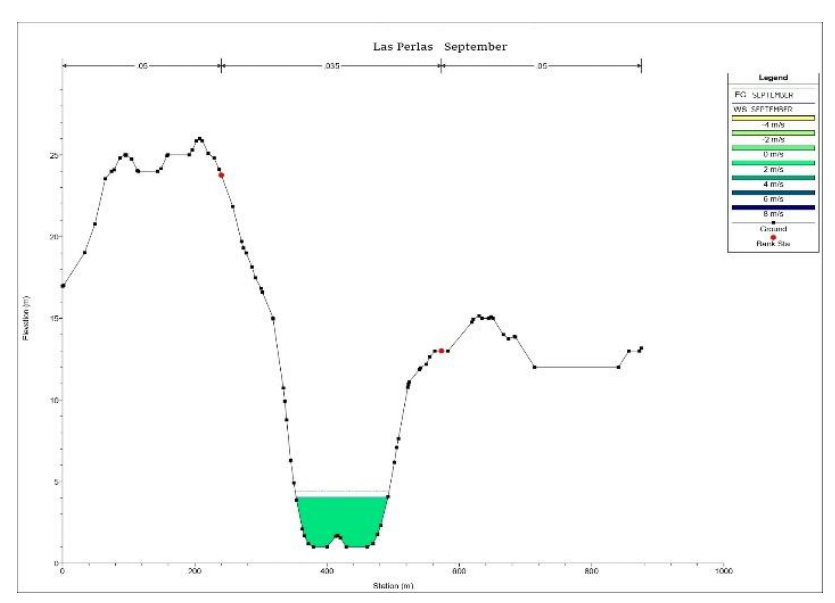

Figura 4 Simulación del río en el sitio las sitio Las Perlas modelado con Hec-Ras. en vista lateral

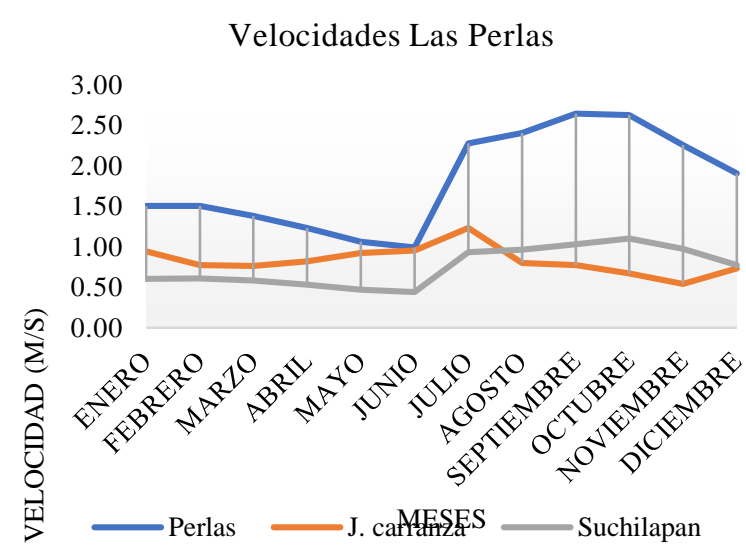

Figura 5 Velocidades en los sitios de estudios calculados con Hec-Ras

En la figura 5 se muestran las velocidades promedio de los 12 meses del año en la sección de los tres sitios de estudio. En la gráfica se puede observar que Las Perlas es el lugar con mayor velocidad, mientras que el sitio Jesús Carranza es el que tiene mayor velocidad a la mitad del año. Aunque el sitio Suchilapan se tiene un mayor caudal, la velocidad es menor durante el inicio de año. En la tabla 1 también muestra el error porcentual que de determina con la siguiente ecuación:

$\%=\left(\frac{Q_{o b s}-Q_{c a l c}}{Q_{o b s}}\right) * 100$

Con la ecuación anterior además de determinar el porcentaje de error, también se puede saber cuándo se están sobre o sub estimando los datos calculados, es decir los valores positivos se están sub estimando los resultados, mientras que los valores negativos se están sobre estimando como se observa en la tabla 1.

\section{Cálculo de potencia}

Teniendo los valores de velocidades de los tres sitios es posible estimar el recurso hidrocinético en cada uno de los mismos. Para ello se ha utilizado la expresión:

$P(t)=C_{p}\left(\frac{1}{2}\right) \rho A v$

Donde,

$P(t)=$ potencia útil obtenida $(\mathrm{kW})$.

$C_{p}=$ coeficiente de potencia. Depende del tipo de turbina a emplear y de la relación existente entre velocidad del flujo y la velocidad de rotación.

$\rho=$ densidad del agua $\left(\mathrm{kg} / \mathrm{m}^{3}\right)$ 
$A=$ el área de la sección transversal opuesta al flujo de la turbina $\left(\mathrm{m}^{2}\right)$.

$v(t)=$ velocidad de la corriente de agua $(\mathrm{m} / \mathrm{s})$.

La ley de Betz propone que el coeficiente teórico de potencia máxima para una turbina hidrocinética es 0.593 . La eficiencia típica para una turbina hidrocinética con bajas pérdidas mecánicas es aproximadamente del 30\%. Para un sistema bien diseñado, el coeficiente de potencia global está entre 0.4 y 0.45 (Yuce y Muratoglu, 2015).

Se determina la potencia con una turbina comercial Thropton Energy Services de eje horizontal donde la velocidad mínima es de 0.5 $\mathrm{m} / \mathrm{s}$ (Sørnes, 2010). El coeficiente de potencia utilizado es de 0.4 .

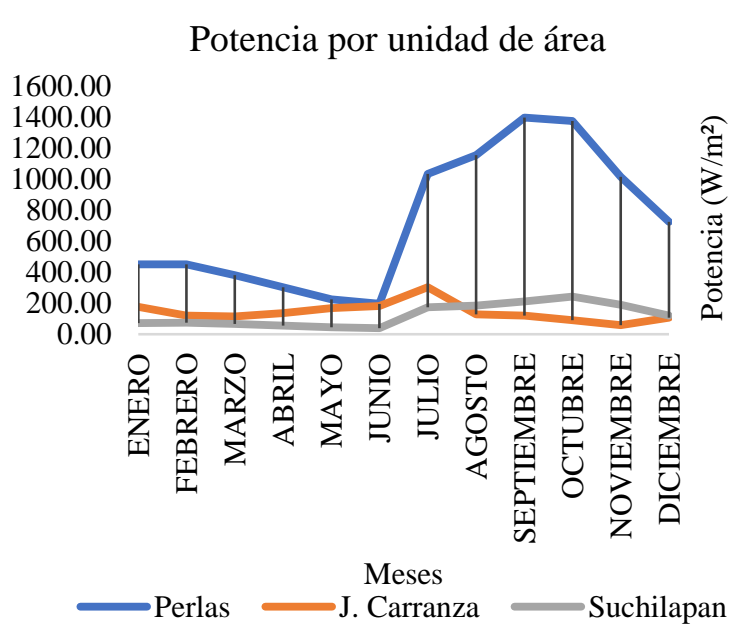

Figura 6 Potencia por unidad de área en los sitios de estudios

\section{Conclusiones}

El sitio de estudio las perlas es el que cuenta con mayor recurso hidrocinético, debido a que tiene el mayor caudal. Aunque en los otros dos sitios se tienen menos caudal, los valores de la velocidad no son inferiores de $0.5 \mathrm{~m} / \mathrm{s}$. Lo que resulta conveniente para el uso de turbina Thropton Energy Services. En el sitio Las Perlas la velocidad a lo largo del año por lo menos es de $1 \mathrm{~m} / \mathrm{s}$ por lo que se podría utilizar otro tipo de turbina. existen tramos donde hay una mayor velocidad nominal suficiente para la producción de energía eléctrica. Al poder estimar los caudales de una forma cercana a los datos medidos, los resultados obtenidos de la estimación del recurso hidrocinético nos da una idea más clara de los lugares donde se puede generar energía eléctrica.
La ventaja que tiene esta forma de generación de energía eléctrica es que se puede realizar a una corta distancia en comparación con la energía minihidráulica, debido a que las turbinas pueden colocarse justo al lado de la comunidad que se pretende beneficiar con este recurso. Unas de las desventajas de la generación eléctrica a través de la energía hidrocinética es que, en el sitio de estudios no cuente con la velocidad nominal mínima para el funcionamiento de las diferentes turbinas que se diseñan para este fin. Existen comunidades río arriba donde se puede estimar la generación de energía eléctrica con la misma metodología que se describe en este trabajo.

\section{Referencias}

(Alaska Satellite Facility, 2011) https://vertexretired.daac.asf.alaska.edu/

Álvarez, E.A.; Rico-Secades, M.; Fernández Suárez, D.F.; Gutiérrez-Trashorras, A.J.; Fernández-Francos, J. Obtaining energy from tidal microturbines: A practical example in the Nalón River. Appl. Energy 2016, 183, 100-112.

Arrieta, E. C., Bedoya, J. A., y Clemente, A. R. (2019). Investigación numérica sobre el uso de álabes multielemento en turbina hidrocinética de eje horizontal. Revista UIS Ingenierías, 18(3), 117-128.

Comisión Nacional del Agua (CONAGUA). Estadística del Agua en México (2018), México.

Comisión Nacional del Agua (CONAGUA). Programa de Medidas Preventivas y de Mitigación de la Sequía (PMPMS) en la Cuenca del río Coatzacoalcos (2014), México.

(Comisión Nacional del Agua (CONAGUA)). ftp://ftp.conagua.gob.mx/ocgc/Publicacion/EST ADISTICAS/Hidrologia/lasperlas.pdf

da Silva P, Blanco C.J.C., Mesquita A.L.A., Brasil Junior A.C.P., de Figueiredo N.M., Macêdo E.N., Assessment of hydrokinetic energy resources downstream of hydropower plants. Renew. Energy, 101 (2017), pp. 1203-1214

Diario Oficial de la Federación (DOF). http://www.dof.gob.mx/nota_detalle.php?codig $\mathrm{o}=5485833 \&$ fecha $=07 / 06 / 2017$ 
Ferreira, J. H. I., Camacho, J. R., Malagoli, J. A., \& Júnior, S. C. G. (2016). Assessment of the potential of small hydropower development in Brazil. Renewable and Sustainable Energy Reviews, 56, 380-387.

\section{INEGI}

https://www.inegi.org.mx/temas/usosuelo/

\section{INEGI}

https://www.inegi.org.mx/temas/edafologia/

Lata G., J. C. (2019). Mejoras al dimensionamiento óptimo de sistemas híbridos con energías renovables. (Tesis Doctoral). Escuela Politécnica Supeiror de Jaén Departamento de Ingeniería Eléctrica, Universidad de Jaén. España.

Motoya R.D, Cuervo F.I., Rico C.A.M. Technical and financial valuation of hydrokinetic power in the discharge channels of large hydropower plants in Colombia: a case study. Renew. Energy, 99 (2016), pp. 136-147

Punys P., Adamonyte I., Kvaraciejus A., Martinaitis E., Vyciene G., Kasiulis E., Riverine hydrokinetic resource assessment. A case study of a lowland river in Lithuania, Renew. Sustain. Energy Rev., 50 (2015), pp. 643-652

Peña G., V. (2013). Diseño de una turbina hidrocinética para aprovechamiento energético de ríos no caudalosos. (Tesis de pregrado) Ingeniería Mecánica Eléctrica. Universidad de Piura. Facultad de Ingeniería. Programa Académico de Ingeniería Mecánica Eléctrica. Piura, Perú.

QGIS Development Team (QGIS). (2018), Geographic Information System Open Source Geospatial Found

Secretaria de Energía (SENER). (2018), Prospectivas de Energías renovables 2018-2032, México.

Secretaria de Energía (SENER). (2017), Prospectivas de Energías renovables 2017-2031, México.

Secretaria de Energía (SENER). (2017b) Mapa de Ruta Tecnológica en energía del Océano, México
Solomon S., Qin D., Manning M., Chen Z., Marquis M., Averyt K.B., Tignor M., Miller H.L., Summary for Policymakers. Climate Change 2007: The Physical Science Basis. Cambridge UniversityPress, Cambridge, Reino Unido y Nueva York, NY, EUA, (2007)

Sørnes K., (2010). Small-scale Water Current Turbines for River Applications. ZERO - Zero Emission Resoruce Organisation

(US Army Corps of Engineers. Hydrologic Engineering Center; 2017) n.d. <http://www.hec.usace.army.mil/software/hecras/ $>$

Yuce M.I., Muratoglu A. Hydrokinetic energy conversion systems: a technology status review Renew. Sustain. Energy Rev., 43 (2015), pp. 7282 